\title{
Risk factors for retinopathy of prematurity: a systematic review
}

\author{
Fatores de risco para retinopatia da prematuridade: uma revisão sistemática

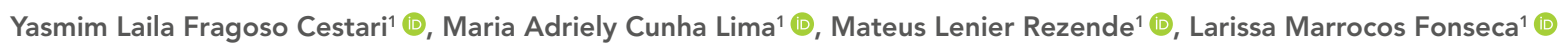

1 Universidade Tiradentes, Aracaju, SE, Brazil

How to cite:
Cestari YL, Lima MA, Rezende ML, Fonseca LM. Risk factors for retinopathy of prematurity: a systematic review. Rev Bras Oftalmol. 2021;80(6):e0052.

https://doi.org/10.37039/1982.8551.20210052

Retinopathyords:

Retinopathy of prematurity; Risk factors; Newborn; Oxygen inhalation therapy; Morbidity

Descritores:

Retinopatia da prematuridade;

Fatores de risco;

Recém-nascido; Oxigenoterapia;

Morbidade

Received on: Feb 19, 2021

Accepted on: Aug 6, 2021

Corresponding author: Maria Adriely Cunha Lima Rua Euclides Paes Mendonça, 957 Mamede Paes Mendonça Zip code: 49509-173 - Itabaiana, SE, Brazil E-mail: mariaadrielycunha@hotmail.com Institution:

Universidade Tiradentes, Aracaju, SE,

Brazil.

Conflict of interest: no conflict of interest.

Financial support: the authors received no financial support for this work.

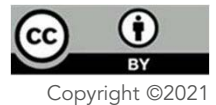

\begin{abstract}
To describe the prevalence of risk factors for retinopathy of prematurity and respective stages. Retrospective data were extracted from original articles addressing risk factors for retinopathy of prematurity retrieved from Scientific Electronic Library Online (SciELO), Virtual Health Library (VHL) and National Library of Medicine - NLM (PubMed) databases. In the initial search, 186 articles were found. Following title and abstract reading and application of inclusion and exclusion criteria, 25 articles were selected for this analysis. Variables of interest varied widely between studies. Gestational age and birth weight were listed as risk factors in all studies. Gender analysis revealed small gender-related differences, since approximately $52.9 \%$ of affected neonates were males and $47.1 \%$ females. As to race/ color, approximately $72.7 \%$ were white, $12 \%$ were brown and $2.7 \%$ were black. However, there is a lack of consensus over the significance of these factors. The study revealed that retinopathy of prematurity is a multifactorial disease primarily associated with prematurity, low birth weight and oxygen therapy. Albeit potentially avoidable and reversible, the incidence of the condition is high. Therefore, further studies along the same lines are needed for deeper understanding of risk factors for or retinopathy of prematurity and mitigation of long-term consequences.
\end{abstract}

\section{RESUMO}

O objetivo deste estudo foi descrever a prevalência dos fatores de risco associados à retinopatia da prematuridade e aos seus estágios. Para isso, foi realizado uma busca nas bases de dados SciELO, VHL e PubMed $\Omega$ de estudos originais que analisavam os fatores de risco para retinopatia da prematuridade foram encontrados. Inicialmente, encontrou-se 186 artigos. Após a leitura dos títulos e dos resumos e de acordo com os critérios de inclusão e de exclusão, foram escolhidos 25 artigos para compor a base de dados deste estudo. Observa-se que houve uma grande diversidade nas variáveis dos estudos. Em relação aos fatores de risco, todos os artigos mencionaram idade gestacional e peso. Ao analisar o sexo, houve uma pequena discrepância, cerca de $52,9 \%$ eram do sexo masculino e $47,1 \%$ do feminino. Em relação à raça/cor, aproximadamente $72,7 \%$ eram brancos, $12 \%$ pardos e $2,7 \%$ pretos. No entanto, não há consenso sobre esses aspectos na literatura. O estudo constatou que a retinopatia da prematuridade é uma doença multifatorial, tendo como principais fatores de risco prematuridade, baixo peso ao nascer e oxigenoterapia. Trata-se de uma doença de alta incidência, apesar de ser evitável e reversível, portanto, pesquisas como esta são essenciais para conhecer os fatores associados e, assim, reduzir as consequências a longo prazo da doença. 


\section{INTRODUCTION}

Retinopathy of prematurity (ROP) is a vasoproliferative ocular disease caused by inappropriate vascularization of the immature retina of preterm newborns (PTNB). ${ }^{(1)}$ The condition was first described by Terry ${ }^{(2)}$ in 1942 and named retrolental fibroplasia. In 1949, Owens and Owens observed the disease could also occur in the postnatal period and, in 1951, Campbell suggested oxygen therapy might be a significant triggering factor. ${ }^{(3,4)}$

Retinopathy of prematurity is a potentially avoidable cause of blindness in children. Approximately 50,000 new cases of ROP are diagnosed annually, primarily in Latin America newborns. ${ }^{(1)}$ In Brazil, 16,00o PTNBs are estimated to develop ROP each year and approximately $10 \%$ of these cases progress to blindness when left untreated. ${ }^{(5)}$ Recent studies indicate a growing number of cases of ROP. This may reflect larger numbers of premature births, financial constraints, delayed referral or unpreparedness of many ophthalmologists and resultant misdiagnosis and lack of appropriate treatment. ${ }^{(1,6)}$

Also important, ROP is a multifactorial disease which may involve the following risk factors: oxygen therapy, intracranial hemorrhage, maternal factors such as multiple pregnancies, diabetes mellitus, advanced maternal age, smoking, iron deficiency, placental detachment and maternal preeclampsia, blood transfusions, septicemia, congenital infections, patent ductus arteriosus, Apgar score lower than 7 at five minutes, small stature for gestational age (GA) and especially prematurity and low birth weight (BW). ${ }^{(7.8)}$ It is estimated that 66 to $68 \%$ of PTNBs weighing less than 1,251 g develop ROP and this percentage rises to $82 \%$ in PTNBs weighing less than 1,000 g. ${ }^{(9)}$

The International Classification of Retinopathy of Prematurity (ICROP) defines ROP according to the anteroposterior location of retinopathy (zones I, II and III), circumferential extension (clock hour distribution or $30^{\circ}$ sectors), severity of abnormal vascular response (stages 1 to 5) and additional signs of severity such as venous dilatation and retinal artery tortuosity, which indicate more aggressive disease (plus disease). This classification is used for recognition of threshold ROP, which is defined as stage 3 plus disease with more than 5 contiguous or 8 cumulative clock hours of fibrovascular proliferation in zones I and II. ${ }^{\left({ }^{10)}\right)}$ The clinical significance of threshold ROP lies in the fact that, in the absence of appropriate treatment, approximately 50\% of cases will progress to retinal detachment and loss of vision..$^{(11)}$

In order to mitigate long-term consequences, Sociedade Brasileira de Pediatria (SBP) recommends binocular indirect ophthalmoscopy between the $4^{\text {th }}$ and $6^{\text {th }}$ weeks of life in newborns (NBs) with BW less than $1.501 \mathrm{~g}$ and/or GA under or equal to 32 weeks. Respiratory distress syndrome or sepsis, blood transfusions, multiple pregnancies and intraventricular hemorrhage are also indications for binocular indirect ophthalmoscopy, since these are thought to be risk factors for threshold ROP development. ${ }^{(12)}$

The traditional treatment of threshold ROP consists of complete destruction of the avascular retina using transpupillary photocoagulation or transscleral cryotherapy. Cases diagnosed in the initial phase of disease and appropriately treated have $41 \%$ less chance of progressing to retinal detachment and 9 to $24 \%$ less chance of progressing to blindness within 5 to 15 years. However, in more than $40 \%$ of affected children, visual acuity in the treated eye is less than 20/200. ${ }^{(9,13)}$

With regard to the pre-threshold stage of the disease, early treatment is only indicated in type 1 pre-threshold ROP (zone I plus with any stage, zone I stage 3 with no plus and zone II stages 2 or 3 plus). Treatment reduces the risk of severe visual impairment and structural damage from $19.5 \%$ to $14.5 \%$ and from $15.6 \%$ to $9.1 \%$ respectively. Still, in spite of laser treatment, affected patients have persistent vascular activity and rates of progression to retinal detachment are high. In patients with type 2 pre-threshold ROP (zone I stage 1 or 2 with no plus or zone II stage 3 with no plus) treatment is not recommended, since spontaneous regression is expected in $52 \%$ of cases. However, these cases may progress to type 1 disease and must therefore be closely monitored. ${ }^{13}$

Given ROP is clinically significant and a public health concern, this review set out to describe the prevalence of risk factors for the condition and respective stages.

\section{METHODS}

This systematic review stemmed from a quantitative, retrospective and documentary research addressing ROP in Brazil. Articles published between 2010 and 2020 were used. Importantly, systematic reviews are defined as secondary studies based on data extracted from primary studies.

The Scientific Electronic Library Online (SciELO), the Virtual Health Library (VHL) and the National Library of Medicine (NLM; PubMed ${ }^{\circledR}$ ) databases were searched using the search terms "retinopathy", "prematurity" and "risk factors". In order to limit the number of articles retrieved, the Boolean operator "AND" and the following filters were applied: articles published in the last 10 
years, studies conducted in Brazil, search terms used in the title or abstract, full text and no language restrictions. Government information, editorials, expert opinions, health guides, Ministry of Health documents, comments, technical and scientific reports were excluded. Articles unrelated to the topic or that did not match the specific purpose of this work were also left out.

A total of 4,348 articles were retrieved in the initial search using the Boolean operator described. Following filter application, 3,407 articles were excluded, and 941 articles retained. Exclusion criteria were then applied, and 86 articles selected for title reading. Of these, 54 articles were extracted for full text reading. The final sample comprised 25 articles, which formed the basis of this analysis (Figure 1).

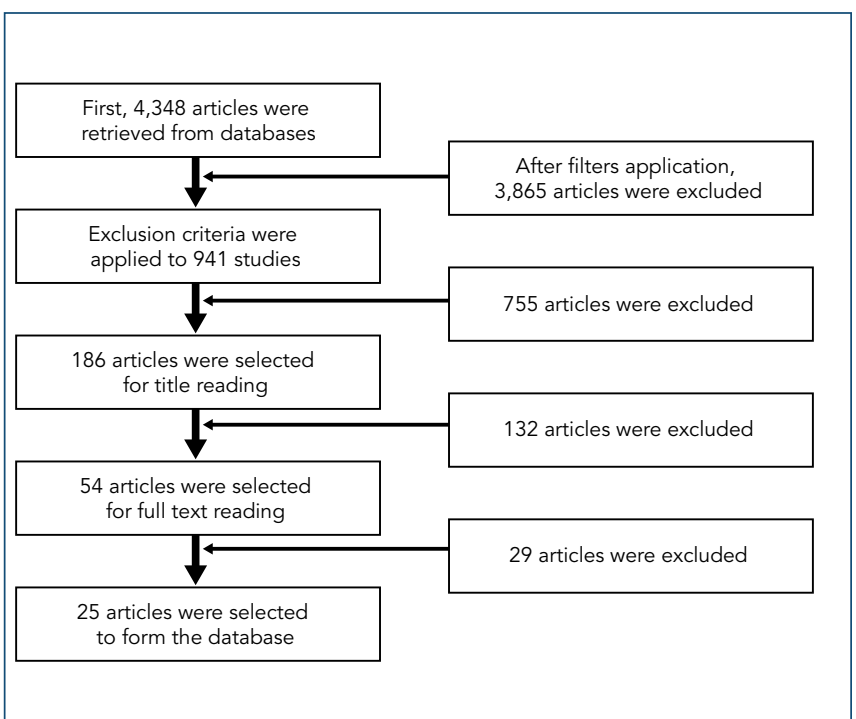

Figure 1. Flow chart showing the article selection process.

Selected articles were reviewed by two independent investigators. In case of mismatches, a third investigator was called upon to determine whether the article in question should be retained in the sample. The Kappa coefficient was used to examine the level of agreement between the investigators - in other words, the ability to measure equal results derived from dependent samples. The kappa coefficient in this study (0.8173) indicated almost perfect agreement (Landis et al.). . ${ }^{(14)}$ Therefore, the analysis was continued (Table 1).

Table 1. Kappa coefficient

\begin{tabular}{|c|c|c|}
\hline & Category $1^{*}$ & Category $2 \dagger$ \\
\hline Category Kappa & 0.8173 & 0.8173 \\
\hline Standard error of category Kappa & 0.0645 & 0.0636 \\
\hline $95 \%$ confidence interval for category Kappa & $\begin{array}{l}\text { Upper: } 0.9438 \\
\text { Lower: } 0.6908\end{array}$ & $\begin{array}{l}\text { Upper: } 0.9417 \\
\text { Lower: } 0.6929\end{array}$ \\
\hline
\end{tabular}

\section{RESULTS}

The number of NBs in articles in this sample totaled 6,897 . Only one article ( $4 \%$ ) failed to describe the number of patients with or without ROP in a sample of 71 NBs. ${ }^{(15)}$ Retinopathy of prematurity affected 2,128 NBs, whereas 4,698 NBs did not develop the disease (30.8\% and 67.2\% respectively).

Most articles included in this study were published in 2019 (28\%), followed by 2010 and 2018 (16\% respectively) (Table 2). No articles published between 2014 and 2020 were selected. In this sample, $36 \%$ of selected articles were cohort studies and approximately 56\% retrospective studies.

Table 2. Type of studies and sample size

\begin{tabular}{|c|c|c|}
\hline Author & $\begin{array}{l}\text { Sample } \\
\text { size }(n)\end{array}$ & Type of study \\
\hline Souza ${ }^{(11)}$ & 50 & $\begin{array}{l}\text { Prospective descriptive and analytical longitudinal } \\
\text { clinical trial for individualized intervention }\end{array}$ \\
\hline Rover et al. ${ }^{(15)}$ & 71 & Retrospective study \\
\hline Portes et al. ${ }^{(16)}$ & 151 & Observational cohort study \\
\hline Schumann et al. ${ }^{(17)}$ & 73 & Retrospective cross-sectional study \\
\hline Shinsato et al..$^{(18)}$ & 70 & Prospective study \\
\hline Tomé et al. ${ }^{(19)}$ & 148 & Retrospective cross-sectional observational study \\
\hline Almeida et al. (20) & 33 & Retrospective cohort study \\
\hline Vieira et al.(21) & 267 & Retrospective study \\
\hline Fortes Filho et al. ${ }^{(22)}$ & 157 & Prospective cohort study \\
\hline Gonçalves et al. ${ }^{(23)}$ & 110 & Longitudinal study \\
\hline Jorge et al. ${ }^{(24)}$ & 232 & Prospective cohort study \\
\hline Moinho et al. ${ }^{(25)}$ & 343 & Observational case-control study \\
\hline Silva et al. ${ }^{(26)}$ & 172 & Retrospective, analytical, case-control study \\
\hline Theis et al. ${ }^{(27)}$ & 320 & Cross-sectional and retrospective study \\
\hline Freitas $^{(28)}$ & 602 & Retrospective cohort study \\
\hline Horewicz et al.(29) & 183 & $\begin{array}{l}\text { Quantitative research approach with descriptive cross- } \\
\text { sectional design }\end{array}$ \\
\hline Martins ${ }^{(30)}$ & 280 & $\begin{array}{l}\text { Observational epidemiological study with case control } \\
\text { design }\end{array}$ \\
\hline Pereira et al..$^{(31)}$ & 296 & Retrospective cross-sectional study \\
\hline Fonseca et al. ${ }^{(32)}$ & 323 & Cohort study \\
\hline Lamy-Filho. ${ }^{(33)}$ & 1.961 & Retrospective cohort study \\
\hline Malheiro et al.(34) & 375 & Retrospective study \\
\hline Okamoto et al. ${ }^{(35)}$ & 58 & Cohort study \\
\hline Pastro et al. ${ }^{(36)}$ & 181 & Retrospective cohort study \\
\hline Xavier et al. ${ }^{(37)}$ & 119 & Cross-sectional, retrospective study \\
\hline Cagliari et al..$^{(38)}$ & 322 & Retrospective study \\
\hline
\end{tabular}

Only two (8\%) of the articles in which the sample was divided into patients with or without ROP did not use ICROP disease classification criteria (severity stages 1 to 5). ${ }^{(31,32)}$ All articles addressed stages 1 and 3 ROP and only one article ${ }^{(22)}$ did not include stage 2 ROP. As to stages 4 and 5 , NBs with stage 4 ROP were investigated in ten articles $(40 \%)^{(16,21,22,24,25,27,30,32,34,38)}$ and NBs with stage 5 ROP in eight articles (32\%). . $16,21,22,27,30,31,32,38)^{2}$

With regard to the number of eyes analyzed and classified according to ROP severity, 4,698 did not have 
ROP, 820 had stage 1 disease and 404 had stage 2 disease (68.1\%, $11.9 \%$ and $5.8 \%$ respectively). Remaining eyes were classified as stage $3(286,4.1 \%)$, stage $4(28,0.4 \%)$ or stage $4(25,0.4 \%)$.

Characteristics of NBs described in studies in this sample are shown in table 3. Variables of interest varied widely between studies, with the exception for the GA and the BW, which were addressed all studies. In some studies, ${ }^{(20)}$ analysis of variables was limited to NBs with ROP.

Gender analysis revealed small gender differences, since approximately $52.9 \%$ of NBs were male and $47.1 \%$ female (1,735 and 1,544 respectively). As to type of pregnancy, approximately $80 \%$ were single and $20 \%$ multiple
(1,656 and 425 respectively). Only four studies contained prenatal care information. In those studies, approximately $73.6 \%,{ }^{(23)} 83.6 \%{ }^{(17)} 89 \%{ }^{(32)}$ and $92.3 \%{ }^{(33)}$ of pregnancies were followed up. Only two articles ${ }^{(17,23)}$ addressed complications during pregnancy or labor. Complication rates in those studies (64.4\% and 38.3\% respectively) were similar to rates reported by Schumann et al. ${ }^{(17)}$. Race/color data were reported in a single article. ${ }^{(29)}$ In that article, approximately $72.7 \%$ of NBs were white, $12 \%$ were brown and $2.7 \%$ were black $(133,22$ and 5 respectively). The color/ race of remaining NBs $(23,12.6 \%)$ was not informed. As to risk factors, GA and BW were mentioned in all studies (Table 4).

Table 3. Sample characteristics

\begin{tabular}{|c|c|c|c|c|c|c|}
\hline Author & $\begin{array}{c}\text { Gender } \\
\text { (male/female) }\end{array}$ & $\begin{array}{l}\text { Gestational age } \\
\text { (weeks) }\end{array}$ & Birth weight (gram) & Gestation & Mode of delivery & Apgar Score \\
\hline Souza(11) & $23 / 27$ & $\begin{array}{c}\text { <30: } 14 \\
\text { 30-32: } 24 \\
>32: 11 \\
\text { Average: } 30.9\end{array}$ & $\begin{array}{l}<1,000: 12 \\
1,000-1,500: 35 \\
>1,500: 3\end{array}$ & $\begin{array}{l}\text { Twin: } 4 \\
\text { Single: } 46\end{array}$ & - & - \\
\hline Rover et al. ${ }^{(15)}$ & $36 / 35$ & Average: 29.4 & $\begin{array}{c}\text { VLBW } \\
\text { AGA: } 50 \text { NBs }\end{array}$ & - & $\begin{array}{l}\text { Vaginal: } 29 \\
\text { Cesarean: } 42\end{array}$ & - \\
\hline Portes et al. ${ }^{(16)}$ & - & Average: 26.62 & Average: 1,109 & - & - & 1st minute average: 5.5 \\
\hline Schumann et al. ${ }^{(17)}$ & $33 / 40$ & Average: 30.5 & $\begin{array}{l}\text { Average: } 1,153 \\
\text { SGA: } 36\end{array}$ & $\begin{array}{l}\text { Prenatal care: } 61 \\
\text { Intercurrence: } 47\end{array}$ & $\begin{array}{c}\text { Vaginal: } 23 \\
\text { Cesarean: } 50 \\
\text { Intercurrence: } 28\end{array}$ & 5th minute average: 8 \\
\hline Shinsato et al..$^{(18)}$ & - & Average: 28.155 & Average: $1,030.935$ & - & - & 1st minute average: 5.2 \\
\hline Tomé et al.(19) & & $\begin{array}{c}<28: 8 \\
\text { 28-32: } 65 \\
31-34: 56 \\
35-36: 17 \\
>36: 3\end{array}$ & $\begin{array}{c}\leq 1,000: 27 \\
1,000-1,500: 115 \\
>1,500: 6\end{array}$ & - & - & $\begin{array}{l}\text { 1st minute } \leq 3: 20 \\
\text { 5th minute } \leq 7: 27\end{array}$ \\
\hline Almeida et al..$^{(20)}$ & - & Average: 30.51 & Average: $1,182.35$ & - & - & - \\
\hline Vieira et al.(21) & $135 / 132$ & Average: 29 & Average: 1,193 & $\begin{array}{l}\text { Twin: } 72 \\
\text { Single: } 195\end{array}$ & $\begin{array}{c}\text { Vaginal: } 69 \\
\text { Cesarean: } 198\end{array}$ & - \\
\hline Fortes Filho et al. ${ }^{(22)}$ & $65 / 92$ & Average: 28.3 & Average: 844,04 & - & Twin: 22 & - \\
\hline Gonçalves et al. ${ }^{(23)}$ & $54 / 56$ & $\leq 32$ & $\leq 1,500$ & $\begin{array}{l}\text { Prenatal care: } 81 \\
\text { Intercurrence: } 79\end{array}$ & $\begin{array}{c}\text { Vaginal: } 72 \\
\text { Cesarean: } 38\end{array}$ & $\begin{array}{c}<7 \\
\text { 1st minute: } 73 \\
\text { 5th minute: } 26\end{array}$ \\
\hline Jorge et al. ${ }^{(24)}$ & $118 / 114$ & Average: 29.4 & Average: $1,219.4$ & $\begin{array}{l}\text { Twin: } 39 \\
\text { Single:193 }\end{array}$ & - & - \\
\hline Moinho et al. ${ }^{(25)}$ & $186 / 157$ & Average: 29.5 & Average: 1,173 & $\begin{array}{c}\text { Twin: } 93 \\
\text { Single: } 250 \\
\text { Complicated: } 215\end{array}$ & - & - \\
\hline Silva et al. ${ }^{(26)}$ & $98 / 74$ & Average: 29.4 & Average: $1,171.9$ & - & - & 1st minute average: 5 \\
\hline Theis et al. ${ }^{(27)}$ & - & $\begin{array}{c}<32: 194 \\
32-37: 115 \\
>37: 11\end{array}$ & $\begin{array}{l}<1,000: 54 \\
\text { 1,000-2,500: } 251 \\
>2,500: 15\end{array}$ & $\begin{array}{l}\text { Twin: } 49 \\
\text { Single: } 271\end{array}$ & - & - \\
\hline Freitas ${ }^{(28)}$ & $302 / 300$ & Average: 29.4 & $<1,000: 148$ & $\begin{array}{l}\text { Twin: } 108 \\
\text { Single: } 494\end{array}$ & - & 1st minute average: 6 \\
\hline Horewicz et al. (29) & $93 / 90$ & $\begin{array}{l}27-31: 66 \\
32-35: 98 \\
>36: 19\end{array}$ & $\begin{array}{c}<970: 19 \\
987-1,950: 140 \\
>1,950: 23\end{array}$ & - & - & - \\
\hline Martins ${ }^{(30)}$ & $132 / 141$ & $\begin{array}{l}\leq 27: 55 \\
>27: 224\end{array}$ & $\begin{array}{c}\leq 1,000: 88 \\
1,000-1,500: 188\end{array}$ & - & - & - \\
\hline Pereira et al. ${ }^{(31)}$ & $152 / 144$ & Average: 32.08 & Average: $1,662.54$ & Prenatal care: 273 & $\begin{array}{c}\text { Vaginal: } 93 \\
\text { Cesarean: } 103\end{array}$ & $\begin{array}{c}\quad<7 \\
\text { 1st minute: } 107 \\
\text { 5th minute: } 30\end{array}$ \\
\hline Fonseca et al..$^{(32)}$ & $55 / 45$ & Average: 28.6 & Average: 1,041 & Prenatal care: 89 & $\begin{array}{l}\text { Vaginal: } 37 \\
\text { Cesarean: } 63\end{array}$ & $\begin{array}{c}<7 \\
\text { 5th minute: } 29\end{array}$ \\
\hline Lamy-Filho. ${ }^{(33)}$ & - & $\leq 35$ & $\leq 2,000$ & - & - & - \\
\hline Malheiro et al. ${ }^{(34)}$ & - & Average: 29.3 & Average: $1,158.7$ & - & - & - \\
\hline Okamoto et al. ${ }^{(35)}$ & $26 / 32$ & 30 & Average: $1,061.8$ & - & - & - \\
\hline Pastro et al. ${ }^{(36)}$ & - & $<37$ & $<2,000$ & - & - & - \\
\hline Xavier et al. .(37) & $54 / 65$ & $\begin{array}{c}23-25: 8 \\
26-29: 19 \\
30-32: 4 \\
>32: 0\end{array}$ & $\begin{array}{l}<1,000: 22 \\
1,000-1,499: 6 \\
1,500-2,500: 3 \\
>2,500: 0\end{array}$ & - & $\begin{array}{l}\text { Vaginal: } 73 \\
\text { Cesarean: } 46\end{array}$ & - \\
\hline Cagliari et al. ${ }^{(38)}$ & $173 / 149$ & Average: 29.5 & Average: $1,181.8$ & - & - & - \\
\hline
\end{tabular}


Table 4. Risk factors for retinopathy of prematurity

\begin{tabular}{|c|c|c|c|}
\hline Risk fator & Scientific evidence & $\begin{array}{l}\text { Lack of scientific } \\
\text { evidence }\end{array}$ & Variable \\
\hline Gestational age & All & - & Smaller \\
\hline Birth weight & All & - & Smaller \\
\hline Oxygen exposure time & $\begin{array}{l}\text { Portes et al. }{ }^{(16)} \text { Schumann } \\
\text { et al. }{ }^{(17)} \\
\text { Shinsato et al. }{ }^{(18)} \\
\text { Souza.111) } \\
\text { Tomé et al. } .^{(19)} \\
\text { Almeida et al. }{ }^{(20)} \\
\text { Vieira et al. } .^{(21)} \\
\text { Moinho et al. }{ }^{(25)} \\
\text { Silva et al. } .^{(26)} \\
\text { Theis et al. }{ }^{(27)} \\
\text { Horewicz et al. }{ }^{(29)} \\
\text { Martins }{ }^{(30)} \\
\text { Okamoto et al. } .^{(35)} \\
\text { Pastro et al. } \\
\text { Xavier et al. }{ }^{(37)} \\
\text { Lamy-Filho. } \\
\text { Cagliari et al. }{ }^{(38)} \\
\text { Fortes Filho et al. }\end{array}$ & - & $\begin{array}{l}\text { Longer } \\
\text { duration } \\
\text { (days) }\end{array}$ \\
\hline $\begin{array}{l}\text { Type of oxygen } \\
\text { exposure }\end{array}$ & $\begin{array}{l}\text { Shinsato et al. }{ }^{(18)} \\
\text { Tomé et al. } .^{(19)} \\
\text { Moinho et al. }{ }^{(25)} \\
\text { Lamy-Filho. } .^{(33)} \\
\text { Pastro et al. }{ }^{\left({ }^{(36)}\right.} \\
\text { Cagliari et al. }{ }^{(38)} \\
\text { Fortes Filho et al. }{ }^{(22) \star}\end{array}$ & $\begin{array}{l}\text { Portes et al. }{ }^{(16)} \\
\text { Martins }^{(30)}\end{array}$ & $\begin{array}{l}\text { Mechanical } \\
\text { ventilation } \\
\text { CPAP } \\
\text { Others }\end{array}$ \\
\hline Apgar 1st minute & $\begin{array}{l}\text { Portes et al. } .^{(16)} \text { Pereira } \\
\text { et al. }{ }^{(31)} \\
\text { Silva et al. }{ }^{(26)} \\
\text { Gonçalves et al. }{ }^{(23)}\end{array}$ & - & Changed \\
\hline Apgar 5th minute & $\begin{array}{l}\text { Schumann et al. }{ }^{(17)} \\
\text { Moinho et al. }{ }^{(25)} \\
\text { Silva et al. }{ }^{(26)} \\
\text { Lamy-Filho. }{ }^{(33)} \\
\text { Gonçalves et al. }{ }^{(23)}\end{array}$ & $\begin{array}{l}\text { Portes et al. }{ }^{(16)} \\
\text { Souza(11) } \\
\text { et al. }{ }^{(31)}\end{array}$ & Changed \\
\hline Sepsis & $\begin{array}{l}\text { Moinho et al. }{ }^{(25)} \\
\text { Theis et al. }{ }^{(27)} \\
\text { Martinss }{ }^{(30)} \\
\text { Xavier et al. }{ }^{(37)} \\
\text { Fonseca et al. } .^{(32)} \\
\text { Gonçalves et al. } .^{(23)}\end{array}$ & $\begin{array}{l}\text { Portes et al. }{ }^{(16)} \\
\text { Shinsato et al. }{ }^{(18)} \\
\text { Souza }\end{array}$ & Presence \\
\hline $\begin{array}{l}\text { Comorbidity/ } \\
\text { respiratory syndromes }\end{array}$ & $\begin{array}{l}\text { Vieira et al. } .^{(21)} \\
\text { Moinho et al. } \\
\text { Theis et al. }{ }^{(27)} \\
\text { Freitas }^{(28)} \\
\text { Lamy-Filho. } .^{(33)} \\
\text { Xavier et al. }{ }^{(37)} \\
\text { Gonçalves et al. }{ }^{(23)}\end{array}$ & $\begin{array}{l}\text { Portes et al. }{ }^{(16)} \\
\text { Shinsato et al. }{ }^{(18)} \\
\text { Martins }{ }^{(30)} \\
\text { Silva et al. }{ }^{(26)}\end{array}$ & Presence \\
\hline $\begin{array}{l}\text { Cardiovascular } \\
\text { comorbidity }\end{array}$ & - & Portes et al. ${ }^{(16)}$ & Presence \\
\hline Digestive comorbidity & - & $\begin{array}{l}\text { Silva et al. }{ }^{(26)} \\
\text { Freitas }{ }^{28)}\end{array}$ & Presence \\
\hline $\begin{array}{l}\text { Hemodynamic } \\
\text { impairment }\end{array}$ & Vieira et al. .211) & - & Presence \\
\hline Use of surfactant & $\begin{array}{l}\text { Vieira et al. }{ }^{(21)} \\
\text { Moinho et al. } \\
\text { Silva et al. } .^{(26)} \\
\text { Lamy-Filho. } \\
\text { Gonçalves et al. }{ }^{(23)}\end{array}$ & $\begin{array}{l}\text { Portes et al. }{ }^{(16)} \\
\text { Martins }{ }^{(30)} \\
\text { Xavier et al. }{ }^{(37)}\end{array}$ & $\begin{array}{l}\text { Higher dose } \\
\text { and longer } \\
\text { use }\end{array}$ \\
\hline $\begin{array}{l}\text { Use of aminophylline } \\
\text { or caffeine }\end{array}$ & Gonçalves et al. ${ }^{(23)}$ & - & - \\
\hline Use of indomethacin & Moinho et al. ${ }^{(25)}$ & $\begin{array}{l}\text { Portes et al. } .^{(16)} \\
\text { Martins } \\
(30)\end{array}$ & $\begin{array}{l}\text { Higher dose } \\
\text { and longer } \\
\text { use }\end{array}$ \\
\hline Use of erythropoietin & Vieira et al. ${ }^{(21)}$ & - & $\begin{array}{l}\text { Higher dose } \\
\text { and longer } \\
\text { use }\end{array}$ \\
\hline Use of diuretic & $\begin{array}{l}\text { Shinsato et al. }{ }^{(18)} \\
\text { Gonçalves et al. }{ }^{(23)}\end{array}$ & Martins ${ }^{(30)}$ & $\begin{array}{l}\text { Higher dose } \\
\text { and longer } \\
\text { use }\end{array}$ \\
\hline $\begin{array}{l}\text { Blood type and RH } \\
\text { factor }\end{array}$ & - & Portes et al..$^{(16)}$ & - \\
\hline Neonatal infections & Theis et al. ${ }^{(27)}$ & $\begin{array}{l}\text { Portes et al. }{ }^{(16)} \\
\text { Okamoto et al. }{ }^{(35)}\end{array}$ & Presence \\
\hline $\begin{array}{l}\text { Intraventricular } \\
\text { hemorrhage }\end{array}$ & $\begin{array}{l}\text { Vieira et al. }{ }^{(21)} \\
\text { Theis et al. }{ }^{(27)} \\
\text { Freitas }{ }^{(28)}\end{array}$ & Souza ${ }^{(11)}$ & Presence \\
\hline $\begin{array}{l}\text { Intracranial } \\
\text { hemorrhage }\end{array}$ & - & $\begin{array}{l}\text { Shinsato et al. } .^{(18)} \\
\text { Moinho et al. }{ }^{(25)} \\
\text { Silva et al al. } \\
\text { Martins }^{(30)}\end{array}$ & $\begin{array}{l}\text { Presence } \\
\text { Moinho et } \\
\text { al. }{ }^{(25)}\end{array}$ \\
\hline
\end{tabular}

\begin{tabular}{|c|c|c|c|}
\hline Risk fator & Scientific evidence & $\begin{array}{l}\text { Lack of scientific } \\
\text { evidence }\end{array}$ & Variable \\
\hline $\begin{array}{l}\text { Intracranial } \\
\text { hypertension }\end{array}$ & Tomé et al. ${ }^{(19)}$ & - & Presence \\
\hline Phototherapy & Martins ${ }^{(30)}$ & - & Longer use \\
\hline Blood transfusions & $\begin{array}{l}\text { Schumann et al. }{ }^{(17)} \\
\text { Shinsato et al. }{ }^{(18)} \\
\text { Tomé et al. }{ }^{(19)} \\
\text { Vieira et al. }{ }^{(21)} \\
\text { Theis et al. }{ }^{(27)} \\
\text { Martins }{ }^{(30)} \\
\text { Lamy-Filho. } .^{(33)} \\
\text { Pastro et al. } .^{(36)} \\
\text { Xavier et al. }{ }^{(37)} \\
\text { Cagliari et al. }{ }^{(38)} \\
\text { Gonçalves et al. } .^{(23)}\end{array}$ & $\begin{array}{l}\text { Souza(11) } \\
\text { Okamoto et al. }{ }^{(35)}\end{array}$ & $\begin{array}{l}\text { Larger } \\
\text { number }\end{array}$ \\
\hline Gender & 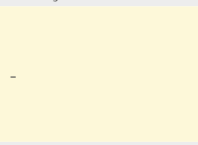 & $\begin{array}{l}\text { Souza(11) } \\
\text { Moinho et al. } \\
\text { Theis et al. } .^{(27)} \\
\text { Freitas } \\
\text { Martins }^{(30)}\end{array}$ & - \\
\hline Mode of delivery & - & $\begin{array}{l}\text { Vieira et al. } \\
\text { Freitas } \\
\text { (21) }\end{array}$ & - \\
\hline Hypoglycemia & - & Okamoto et al. ${ }^{(35)}$ & \\
\hline Jaundice & Xavier et al. ${ }^{(37)}$ & $\begin{array}{l}\text { Silva et al. }{ }^{(26)} \\
\text { Okamoto et al. }{ }^{(35)}\end{array}$ & - \\
\hline $\begin{array}{l}\text { Gestational } \\
\text { complications }\end{array}$ & - & $\begin{array}{l}\text { Moinho et al. }{ }^{(25)} \\
\text { Okamoto et al. }{ }^{(35)}\end{array}$ & Presence \\
\hline Twin pregnancy & Jorge et al.(24) & $\begin{array}{l}\text { Souza }{ }^{(11)} \\
\text { Moinho et al. }{ }^{(25)} \\
\text { Theis et al. }{ }^{(27)}\end{array}$ & - \\
\hline $\begin{array}{l}\text { Patent ductus } \\
\text { arteriosus }\end{array}$ & $\begin{array}{l}\text { Tomé et al. }{ }^{(19)} \\
\text { Moinho et al. }{ }^{(25)} \\
\text { Theis et al. }{ }^{(27)} \\
\text { Xavier et al. }{ }^{(37)} \\
\text { Fonseca et al. } .^{(32)}\end{array}$ & Martins ${ }^{(30)}$ & Presence \\
\hline
\end{tabular}

CPAP: continuous positive airway pressure.

\section{DISCUSSION}

Prematurity is a common cause neonatal disorders due to immaturity. In the majority of cases, preterm babies are born with low weight and require hospitalization and interventions such as oxygen therapy, blood transfusion and neonatal Intensive Care Unit (NICU) procedures. ${ }^{(39)}$ Hence the implementation and refinement of neonatal care techniques aimed at enabling the survival of increasingly premature babies. However, these advanced techniques may contribute to the development of ROP, a major cause of visual impairment or blindness in babies born prematurely. ${ }^{(39,40)}$

In this study, the prevalence of retinopathy was approximately $32.3 \%$. Similar prevalence $(29.9 \%)$ has been reported in prior Brazilian studies. ${ }^{(41)}$ Disease classification analysis revealed that most NBs $(11.9 \%)$ had stage 1 ROP, followed by stages 2, 3, 4 and 5 (5.8\%, 4.1\%, 0.4\% and $0.4 \%$ respectively). Likewise, in a Brazilian study by Torigoe, ${ }^{(40)} 12.17 \%$ of newborns had stage 1 ROP, $8.9 \%$ progressed to stage 2, 5.04\% reached stage 3 and $2.08 \%$ developed stage 3 threshold disease, the most severe form of ROP reported during the follow-up period in that study. The high prevalence and severity of ROP emphasizes the need to discuss related risk factors.

Birth weight and GA are inversely related to the incidence of ROP. ${ }^{(42)}$ Severe ROP is more common in newborns 
born with GA $<28$ weeks or BW $<1,000$ g, with few cases reported among newborns with BW over 1,500 g or GA over 32 weeks. ${ }^{(5)}$

In a sample comprising 1,070 NBs examined by Reisner et al.,(43) ROP prevalence rates of $21 \%$ and $72 \%$ were reported in neonates weighing less than 1,500 $\mathrm{g}$ and 1,000 g respectively. In turn, the relationship between GA and ROP can be explained by the fact that retinal blood vessels reach the periphery of the nasal retina in the eighth month of pregnancy and the temporal retina at term. Therefore, the preterm peripheral retina is avascular at birth. Following premature birth, normal retinal vascular development is interrupted and part of retinal blood vessels are obliterated. ${ }^{(39)}$

The last classic risk factor for ROP is oxygen therapy, which is strongly related to the etiology of the disease. In preterm nenonates submitted to hyperoxia in the first hours or days of life, vascular endothelium growth factor (VEGF) production is inhibited. As a consequence, new blood vessel formation is interrupted and existing vessels are obliterated. Exposure of preterm babies to the natural environment without supplemental oxygen combined with the high metabolic needs of the developing eye create hypoxic conditions in the peripheral retina, triggering large scale VEGF production and leading to the formation of new, but abnormal (i.e., dilated and tortuous) vessels. (44,45) Oxygen therapy induces vasoconstriction in the immature retina and areas of vascular occlusion may result if oxygenation is maintained. ${ }^{(46)}$

However, Jorge et al. ${ }^{(24)}$ and Malheiro et al. ${ }^{(34)}$ did not include oxygen therapy in their investigations of risk factors for ROP development, nor did Freitas ${ }^{(28)}$ or Pereira et al. ${ }^{(31)}$ Lack of sufficient data on oxygen concentrations employed and duration of oxygen therapy in medical records may explain why oxygen therapy was not a statistically significant factor in this analysis.

Several studies describe ROP as a multifactorial condition. ${ }^{(42)}$ Therefore, maternal factors, fluctuating oxygen levels in the first weeks of life, mechanical ventilation, intracranial hemorrhage, blood transfusions, sepsis, congenital infections, patent ductus arteriosus, Apgar score lower than 7 at five minutes, short stature for GA and prematurity and low birth weight in particular may be implicated in ROP etiology. Twin pregnancy, intraventricular hemorrhage, phototherapy, apnea and anemia are other potential etiological factors. ${ }^{(47,48)}$

Anemia and erythropoietin administration may also be risk factors for ROP. Anemia of prematurity tends to be associated with low reticulocyte counts and defective erythropoietin production. Given anemic states may lower oxygen saturation to a critical level, preterm newborns often require blood transfusions. The role of blood transfusions in ROP development in newborns weighing less than $1250 \mathrm{~g}$ at birth has been addressed in several studies and the use of recombinant human erythropoietin in an effort to lessen the need of blood transfusions in preterm newborns has been suggested. However, cumulative recombinant erythropoietin exposure (total 6-week dose) has recently been associated with an increased risk of ROP in preterm neonates. ${ }^{(5)}$

Gender and race/color are other potential newborn-related risk factors for ROP. In this study $52.9 \%$ of the NBs were males, approximately $72.7 \%$ were white, $12 \%$ were brown and were $2.7 \%$ black. However, data regarding the relationship between race/color and ROP development are conflicting. According to Sola et al., ${ }^{(49)}$ male gender and white skin color account for two out of four major risk factors for ROP. Delport et al. ${ }^{(50)}$ reported that the incidence of ROP appears to be slightly lower in black neonates relative to white neonates $(3.2 \%$ and $7.4 \%$, respectively). In turn, in the study by Fortes Filho et al., (5) female gender was implicated.

On the maternal side, hypertensive disorders of pregnancy (HDP), diabetes mellitus and medication may play a role in ROP. Advanced maternal age and smoking are risk factors for preterm birth and low BW, respectively. Hypertensive disorders of pregnancy are often associated with perinatal morbidities and higher levels of anti-angiogenic factors such as sFlt-1 (soluble fms-like tyrosine kinase-1), a VEGF antagonist. As to diabetes, direct (e.g., increased retinal VEGF levels in response to hyperglycemia) and indirect (e.g., associations with respiratory distress syndrome) impacts on ROP development have been reported. ${ }^{(51)}$

Importantly, risk factors differed between univariate and multivariate analysis in some studies. In the study by Silva et al., ${ }^{(26)}$ different from GA and duration of oxygen therapy, birth weight, Apgar score at 1 and 5 minutes, need of surfactant and jaundice (time of onset and indirect bilirubin level) were not independent risk factors for ROP development in multivariate logistic regression analysis. However, the latter variables were significant risk factors in univariate analysis. Also, in one study ${ }^{(29)}$ caffeine was a protective factor against $\mathrm{ROP}$, whereas another study ${ }^{(31)}$ described resuscitation in the delivery room as being protective.

Unlike other selected articles, Rover et al. ${ }^{(15)}$ examined the consequences of ROP and described relationships between growth deficit in the first and second trimesters of life and very low BW, since BW interferes with weight gain 
and height in preterm newborns. In NBs up to 3 months of age, ROP increases the risk of failure to thrive and is associated with a 7.2-fold higher risk of weight deficit and a 15-fold higher risk of height deficit.

Retinopathy of prematurity is a multifactorial disease associated primarily with low BW and oxygen therapy. Findings of this study and existing incidence data suggest that, in spite of the avoidable and reversible nature of ROP, risk factors for this condition deserve further investigation in order to mitigate long-term consequences. The significance of early diagnosis and appropriate treatment in the initial phase of the disease in order to prevent progression to visual impairment or blindness in preterm newborns has been emphasized.

\section{REFERENCES}

1. Zin A, Florêncio T, Fortes Filho JB, Nakanami CR, Gianini N, Graziano RM, et al; Brazilian Society of Pediatrics, Brazilian Council of Ophthalmology and Brazilian Society of Pediatric Ophthalmology. [Brazilian guidelines proposal for screening and treatment of retinopathy of prematurity (ROP)]. Arq Bras Oftalmol. 2007;70(5):875-83. Portuguese.

2. Terry TL. Extreme prematurity and fibroblastic over-growth of persistent vascular sheath behind each crystalline lens. Am J Ophthalmol. 1942;25:203-4.

3. Campbell K. Intensive oxygen therapy as a possible cause of retrolental fibroplasia; a clinical approach. Med J Aust. 1951;2(2):48-50.

4. Owens WC, Owens EU. Retrolental fibroplasia in premature infants. Am J Ophthalmol. 1949; 32(1):1-21.

5. Fortes Filho JB, Eckert GU, Procianoy L, Barros CK, Procianoy RS. Incidence and risk factors for retinopathy of prematurity in very low and in extremely low birth weight infants in a unit-based approach in southern Brazil. Eye (Lond). 2009;23(1):25-30.

6. Fortes Filho JB, Eckert GU, Valiatti FB, Costa MC, Bonomo PP, Procianoy RS. Prevalência e fatores de risco para a retinopatia da prematuridade: estudo com 450 pré-termos de muito baixo peso. Rev Bras Oftalmol. 2009;68(1):22-9.

7. Bashinsky AL. Retinopathy of prematurity. N C Med J. 2017;78(2):124-8.

8. Pinheiro AM, Silva WA, Bessa CG, Cunha HM, Ferreira MA, Gomes AH. Incidência e fatores de risco da retinopatia da prematuridade no Hospital Universitário Onofre Lopes, Natal (RN) - Brasil. Arq Bras Oftalmol. 2009;72(4):451-6.

9. Multicenter trial of cryotherapy for retinopathy of prematurity. Preliminary results. Cryotherapy for Retinopathy of Prematurity Cooperative Group. Arch Ophthalmol. 1988;106(4):471-9.

10. International Committee for the Classification of Retinopathy of Prematurity. The International Classification of Retinopathy of Prematurity revisited. Arch Ophthalmol. 2005 [cited 20212 Aug];123(7):991-9. Available from: https:// portaldeboaspraticas.iff.fiocruz.br/wp-content/uploads/2018/01/esa40009.pdf

11. Souza RA. Retinopatia da prematuridade: incidência, detecção e conduta em hospital de referência no Distrito Federal. Brasília, DF: Universidade de Brasília, Faculdade de Ciências da Saúde; 2010.

12. Grupo Retinopatia da Prematuridade Brasil. Relatório do IV Workshop Retinopatia da Prematuridade. Sociedade Brasileira de Pediatria; Rio de Janeiro; 2002

13. Conselho Brasileiro de Oftalmologia; Sociedade Brasileira de Pediatria; Sociedade Brasileira de Retina e Vítreo; Sociedade Brasileira de Oftalmologia Pediátrica. Retinopatia da prematuridade. São Paulo: CBO; 2011.

14. Landis JR, Koch GG. The measurement of observer agreement for categorical data. Biometrics. 1977;33(1):159-74
15. Rover MM, Viera CS, Silveira RC, Guimarães AT, Grassiolli S. Risk factors associated with growth failure in the follow-up of very low birth weight newborns. J Pediatr (Rio J). 2016;92(3):307-13.

16. Portes $A L$, Baraúna $H$, Jeveaux G, Monteiro ML. Perfil clínico e epidemiológico de recém-natos prematuros com muito baixo peso no Rio de Janeiro: estudo de 152 pacientes. Rev Bras Oftalmol. 2010;69(6):38994.

17. Schumann RF, Barbosa AD, Valete CO. Incidência e gravidade da retinopatia da prematuridade e sua associação com morbidade e tratamentos instituídos no Hospital Universitário Antonio Pedro, entre 2003 a 2005. Arq Bras Oftalmol. 2010;73(1):47-51.

18. Shinsato RN, Paccola L, Gonçalves WA, Barbosa JC, Martinez FE, Rodrigues $\mathrm{ML}$, et al. Frequência de retinopatia da prematuridade em recém-nascidos no Hospital das Clínicas da Faculdade de Medicina de Ribeirão Preto da Universidade de São Paulo. Arq Bras Oftalmol. 2010;73(1):60-5.

19. Tomé VA, Vieira JF, Oliveira LB, Pinto RM, Abdallah VO. Study of retinopathy of prematurity in a university hospital. Arq Bras Oftalmol. $2011 ; 74(4): 279-82$.

20. Almeida TS, Souza EV, Campos PH, Oliveira LS, Cunha RD. Incidência de Retinopatia da Prematuridade no Hospital Universitário da Universidade Federal de Santa Catarina entre 2010 e 2011. Arq. Catarin. Med. 2012;41(3):44-8

21. Vieira BC, Nascimento B, Ribeiro I, Carvalho R, Martins JN. Resultados de 12 anos de rastreio da retinopatia da prematuridade no Hospital Pedro Hispano. Oftalmologia. 2013;37(3):199-204.

22. Fortes Filho JB, Fortes BG, Tartarella MB, Procianoy RS. Incidence and main risk factors for severe retinopathy of prematurity in infants weighing less than 1000 grams in Brazil. J Trop Pediatr. 2013;59(6):502-6.

23. Gonçalves E, Násser LS, Martelli DR, Alkmim IR, Mourão TV, Caldeira AP, et al. Incidence and risk factors for retinopathy of prematurity in a Brazilian reference service. Sao Paulo Med J. 2014;132(2):85-91.

24. Jorge EC, Marcon AR, Puerta NA. Retinopatia da prematuridade no Hospital da Faculdade de Medicina de Botucatu - Unesp. Rev Ciênc Ext. 2015;11(3):63-72

25. Moinho R, Morais S, Monteiro M, Mimoso G. Retinopatia da prematuridade numa unidade de cuidados intensivos neonatais: experiência de oito anos. Acta Pediatr Port 2015:46:198-204.

26. Silva FC, Falco HC, Silva FG, Carvalho PK. Retinopathy of prematurity: perinatal risk factors. Semina Ciênc Biol Saude. 2016;37(1):3-14.

27. Theis MB, Junior Grumann A, Rodrigues MR. Perfil epidem iológico dos recém-nascidos prematuros com retinopatia da prematuridade no Hospital Regional de São José Dr. Homero de Miranda Gomes. Rev Bras Oftalmol. 2016;75(2):109-14.

28. Freitas AM. Incidência e fatores de risco para retinopatia da prematuridade em um Serviço de Referência no Rio Grande do Sul. Porto Alegre. Tese Universidade Federal de Ciências da Saúde; 2017.

29. Horewicz VC, Viera CS, Pastro J, Toso BR. The profile of newborns in the ophthalmology service for the control of retinopathy of prematurity. Rev Enferm UFSM 2018;8(4):744-57.

30. Martins VS. Estudo dos fatores de risco para o desenvolvimento da retinopatia da prematuridade em recém nascidos prematuros e de muito baixo peso [tese]. Palhoça: Universidade do Sul de Santa Catarina; 2018.

31. Pereira R, Getirana RS, Uchimura TT, Corrêa DA, Previdelli I, Barili E. Fatores associados e predição da retinopatia da prematuridade. Cad Saúde Colet. 2018;26(1):70-5.

32. Fonseca LT, Senna DC, Eckert GU, Silveira RC, Procianoy RS. Association between human breast milk and retinopathy of prematurity. Arq Bras Oftalmol. 2018;81(2):102-9

33. Lamy-Filho F, Marques PF, Lamy ZC, Costa EP, Mendes RM, Bastos AA, et al Incidence and factors associated a retinopathy of prematurity: experience after applied screening program. Rev Pesq Saúde. 2019;20(1):7-10.

34. Malheiro L, Falcão I, Neiva L, Almeida A, Maia S, Miranda V, et al. Aplicação do modelo WINROP no rastreio de Retinopatia de Prematuridade (ROP) numa amostra de prematuros portugueses. Rev Bras Oftalmol. 2019;78(1):30-6. 
35. Okamoto TC, Assman R, Neto Olderbug C, Nisihara R. Retinopatia da prematuridade: análise de uma tentativa de redução de danos. Rev Bras Oftalmol. 2019;78(2):117-21.

36. Pastro J, Toso BR. Influência do oxigênio no desenvolvimento de retinopatia da prematuridade. Rev Bras Enferm. 2019;72(3):623-30.

37. Xavier MSLRA, Santos TMSA. Ocorrência de retinopatia da prematuridade em recém-nascidos de muito baixo peso em maternidade de referência terciária no município de Fortaleza - CE. Ver Med UFC. 2019; 59 (4): 7-13.

38. Cagliari PZ, Lucas VC, Borba IC, Leandro DM, Gascho CL, Veras TN, et al. Validation of ROPScore to predict retinopathy of prematurity among very low birth weight preterm infants in a southern Brazilian population. Arq Bras Oftalmol. 2019;82(6):476-80.

39. Yogui JO. A ocorrência e os fatores associados à retinopatia da prematuridade (ROP) em recém-nascidos prematuros atendidos no ambulatório de follow-up de prematuro do HU-UFJF. Juiz de Fora. Dissertação [Mestrado] - Universidade Federal de Juiz de Fora; 2015.

40. Torigoe AM. Retinopatia da prematuridade: incidência, detecção e fatores relacionados. Campinas. Tese - Universidade Estadual de Campinas: 2005.

41. Graziano RM. Retinopatia da prematuridade: contribuição ao estudo da ocorrência e análise dos fatores de risco. São Paulo. Tese - Faculdade de Medicina, Universidade de São Paulo; 1994.

42. Lermann VL, Fortes JB, Procianoy RS. Prevalência de retinopatia da prematuridade em recém-nascidos de muito baixo peso. J Pediatr. $2006 ; 82(1): 27-32$
43. Reisner SH, Shohat M, Krikler R, Nissenkrn I, Ben Sira I. Retinopathy of prematurity: incidence and treatment. Arch Dis Child. 1985;60:698-701.

44. Good WV, Hardy RJ, Dobson V, Palmer EA, Phelps DL, Quintos M, et al; Early Treatment for Retinopathy of Prematurity Cooperative Group. The incidence and course of retinopathy of prematurity: findings from the early treatment for retinopathy of prematurity study. Pediatrics. 2005;116(1):15-23.

45. Graziano RM, Leone CR. Problemas oftalmológicos mais freqüentes e desenvolvimento visual do pré-termo extremo. J Pediatr (Rio J). 2005;81(1 Suppl 1):S95-S100.

46. Sira BI. Nissenkorn I, Kremer I. Retinopathy of prematurity. Surv Ophthalmol. 1988;33(1):1-16

47. Hakeem AH, Mohamed GB, Othman MF. Retinopathy of prematurity: a study of prevalence and risk factors. Middle East Afr J Ophthalmol. 2012;19(3):289-94.

48. Huang HM, Lin SA, Chang YC, Kuo HK. Correlation between periventricular leukomalacia and retinopathy of prematurity. Eur J Ophthalmol. 2012;22(6):980-4

49. Sola A, Chow L, Rogido M. Retinopatía de la prematuridad y oxigenoterapia: una relación cambiante. Ann Pediatr. 2005;62(1):48-63.

50. Delport SP, Swanepoel JC, Odendaal PJ, Roux P. Incidence of retinopathy of prematurity in very-low-birth-weight infants born at Kalafong Hospital, Pretoria. S Afr Med J. 2002;92(12):986-90.

51. Kim SJ, Port AD, Swan R, Campbell JP, Chan RV, Chiang MF. Retinopathy of Prematurity: A Review of Risk Factors and their Clinical Significance. Surv Ophthalmol. 2018;63(5): 618-37. 